\title{
Overexpression of GRP78 receptor and its Chemical Biology in Cancer and Autoimmune Diseases: High risk for COVID 19?
}

${ }^{5}$ Arup Kumar Banerjee, 1,2Feroza Begum, 2,4Amit Kumar Srivastava*, 2,3Prem Prakash Tripathi* ${ }^{*}, 2$ Upasana Ray ${ }^{*}$

\section{Affiliations:}

1. Infectious Biology and Immunology Division, CSIR-Indian Institute of Chemical Biology, 4, Raja S.C., Mullick Road, Jadavpur, Kolkata-700032, West Bengal, India.

2. Academy of Scientific and Innovative Research (AcSIR), Ghaziabad- 201002, India

3. Cell Biology \& Physiology Division, CSIR-Indian Institute of Chemical Biology, 4, Raja S.C., Mullick Road, Jadavpur, Kolkata-700032, West Bengal, India.

4. Cancer Biology and Inflammatory Disorder Division, CSIR-Indian Institute of Chemical Biology, 4, Raja S.C., Mullick Road, Jadavpur, Kolkata-700032, West Bengal, India.

5. Department of Biochemistry, North Bengal Medical College and Hospital, Sushrutanagar, Siliguri-734012, West Bengal, India

\section{* Corresponding Authors}

\section{Abstract}

ACE2 receptor has been the focus of scientific community as receptor of COVID 19 and is been explored for therapeutic targeting. Recently, another receptor for COVID 19 was reported, the Glucose Regulated Protein 78 (GRP78). GRP78, a heat shock protein is an endoplasmic reticulum (ER) stress response protein. In cancer and certain autoimmune diseases there are reports of upregulation of GRP78. In breast cancer, prostate cancer, melanomas and malignant gliomas GRP78 overexpresses. Chemoresistance has been linked to upregulated GRP78. So, cancer patients might be at increased risk of COVID 19 infection owing to receptor abundance. We propose that tumour associated endothelial cells having upregulated GRP78 might also participate in dissemination of the virus. GRP78 also gets upregulated in rheumatoid arthritis and inflammatory bowel disease (IBD). Thus, both cancer patients as well as autoimmune diseases (Rheumatoid arthritis and IBD) should be considered as highrisk groups for COVID 19.

\section{Introduction}

Novel Coronavirus COVID 19 outbreak turned pandemic recently late in 2019 and continues to kill thousands worldwide. This virus is a close relative of the SARS family of viruses and share many molecular similarities with the other members. COVID 19 is a member of Betacoronaviruses and is the seventh member amongst the human coronaviruses.

Receptor mediated endocytosis is the first step of the life cycle of this virus. This process involves receptor attachment, binding and endocytosis to execute cellular entry. All Coronaviruses including COVID 19 have Spike (S) protein on the virion surface. This is one of the structural proteins of the virus and play the key role in cellular entry [1]. $S$ protein is present on the outer membrane of the virus particle in 
the form of homotrimer. Each monomer of $S$ protein contains $S 1$ and S2 domains. While S1 mediates receptor binding, S2 helps in fusion process.

For a virus to enter its host cell, the primary requirement is host cell receptor that would allow virus attachment and entry. Host cell receptors thus determine virus tropism i.e. the variety of cell types that the virus can invade. Recently a correlation with angiotensin-converting enzyme 2 (ACE2) receptor and COVID 19 infection was established [1] and ACE 2 receptor is considered to be the key receptor that this virus uses to invade human cells. In this context individuals with pre-existing conditions like Diabetes or hypertension have been categorized into high risk groups for COVID 19 and are thought to be prone to increased mortality and morbidity due to this virus. Thus, the scientific community started targeting virus-ACE 2 association for drug designing and therapeutic interventions.

While everyone was focussing on ACE2, Ibrahim et al has recently published an article in the Journal of Infection (was available online on 10 March 2020) where the group proposed Glucose Regulated Protein Receptor (GRP78) as another potential receptor for COVID 19 [2]. This was the first report of another possible receptor and thus also another mode of entry. In this study the authors have also dissected the putative binding sites of the receptor and the Spike protein. Four regions of the spike protein were checked for binding potential with the GRP78 receptor and it was concluded that mainly two of the tested regions C391-C525 and C480-C488 showed favourable affinities. Among these two areas the authors proposed the later to be the key site for spike-GRP78 interaction. This binding site of GRP78 overlaps with the ACE 2 binding site although the exact residues involved in receptor specific interactions might be little different.

GRP 78 is a $78 \mathrm{kDa}$ protein also known as BiP and is member of the heat shock protein (Hsp) 70 family. This protein has many functions in maintaining cell viability and is upregulated under ER (endoplasmic reticulum) stress and disbalance in calcium homeostasis. Under cellular stress, GRP78 mRNA gets translated by IRES (Internal Ribosome Entry Site) mediated translation which is a cap independent mode of translation. Viral infections like herpes simplex virus type 1 have been shown to trigger IRES mediated translation of GRP 78. [3]. Although GRP78 is mainly an ER associated protein, under various conditions it is also expressed in cytosol, nucleus, mitochondria or plasma membrane [4].

Thus, under physiological conditions when GRP78 expression gets upregulated, conditions might potentially be more favourable for COVID 19 infection. In this article we have highlighted diseases where GRP78 upregulation has been reported and we propose to categorize these diseases as high-risk groups. These groups would be additional to the already believed high risk categories i.e. diabetes and hypertension. Presence of both ACE 2 and upregulated GRP 78 could possible put these additional high-risk categories into very high-risk types.

\section{GRP78 and Cancer connection}

In past there have been evidences about specific upregulation of cell surface GRP78 in cancer cells and its role in stem cell regeneration and tumorigenesis. GRP78 plays 
crucial roles in cancer cell chemoresistance and survival. It has also been linked to angiogenesis and metastasis. In case of some cancers like prostate cancer[5,6], breast cancer [7,8] and melanoma [9] there have been reports of abnormal overexpression of GRP78 and this has been correlated with chemoresistance and recurrence [6]. One of the most chemo resistant tumours are the malignant gliomas. In normal human brain GRP78 are expressed at very low levels. However, in malignant gliomas, GRP78 levels increase [10]. A knockdown of GRP78 in glioma cells lead to increased chemotherapy induced cell death/ apoptosis [10] showing that GRP78 has pro-chemoresistance property. GRP78 also have pro-tumorigenic property with respect to tumour associated endothelial cell growth and their chemoresistance. These cells were also seen to exhibit upregulated GRP78 compared with the endothelial cells associated with non-malignant tissues [11]. Since, GRP78 have also been found to get upregulated in tumour associated endothelial cells, it can be postulated that a virus that uses GRP78 as receptor should potentially be able to enter the tumour associated endothelial cells as well (Figure 1). The cellular machinery of endothelial cells might or might not be compatible for virus replication. However, if a virus enters endothelial cells and replicates, it can possibly travel to other tissues and organs via endothelial route i.e. either the tight junctions. This would offer the virus chance to travel to various tissue types and infect those where it gets compatible receptors (Figure 2).

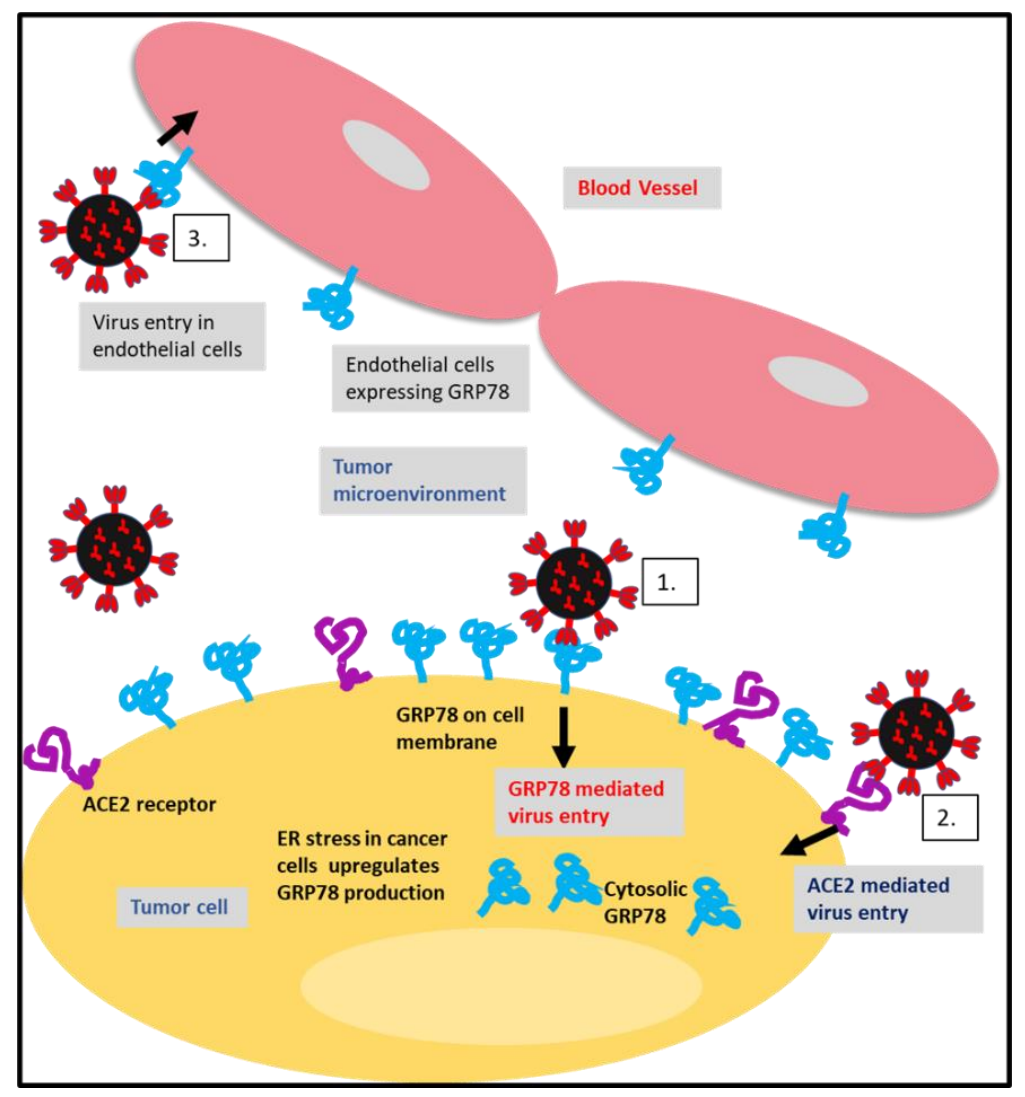

Figure 1 COVID 19 in tumour microenvironment: Tumor/ Cancer cells express high levels of GRP78. These cells may also express normal levels of ACE2 receptor. COVID 19 thus has two options to enter the tumour cell 1. Upregulated GRP78 on cell membrane and 2. ACE2 receptor. Tumour associated endothelial cells also express GRP78 and thus this is yet another mode of virus infection (3). 
The presence of overexpressed GRP78 in certain types of cancer [11-17] and in tumour associated endothelial cells make these susceptible to COVID 19 infection (Figure 1 and 2) and thus might be considered as high-risk categories.

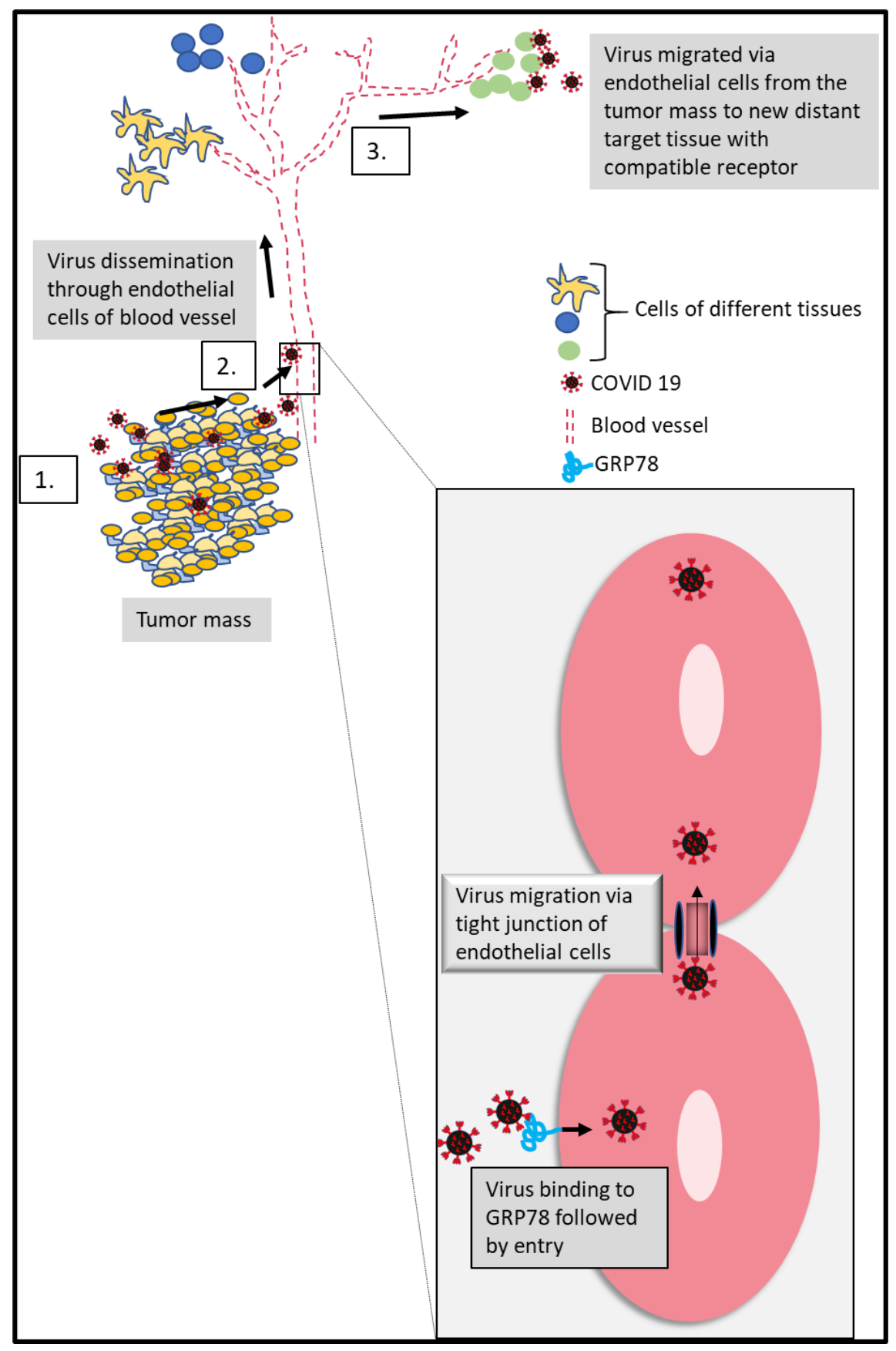

Figure 2 COVID 19 dissemination from tumor mass to distant tissues via endothelial route: This figure shows a hypothetical dissemination route of COVID19. From metastatic tumor (1) mass, the virus could enter the tumor associated endothelial cells (2) which express elevated GRP78 on cell surface. After gaining entry, the virus might replicate and progeny virus particles might travel from one endothelial to another via tight junctions and eventually reach distant tissues (3). Wherever the virus gets compatible receptor/s it can potentially infect new target tissues and proliferate. 


\section{GRP 78 and Autoimmune Diseases}

GRP78 upregulation has also been reported under ER stress in few autoimmune diseases for example Rheumatoid Arthritis (RA). In RA, there is expansion of synovium which contains the synoviocytes, the cell population primarily participating in inflammatory process. It has been shown that in RA, cell surface GRP78 gets upregulated in the synoviocytes and synovial macrophages [18] and promote synoviocyte proliferation. Upregulated surface GRP78 in both synoviocytes as well as synovial macrophages are thus prone to COVID 19 infection. Synovial tissue resident macrophages form a protective shield around the joint. However, this shield cracks open in RA allowing infiltrating macrophages that are the mediators of inflammation. If synovial macrophages with upregulated GRP78 gets infected with COVID 19, viral pathogenic affect might destroy the protective shield further thereby allowing more inflammation and disease severity.

Inflammatory Bowel Disease (IBD) is another autoimmune disease where increased production of GRP78 has been reported and has crucial role in progression of IBD. GRP78 is also secreted out in exosomes in IBD and this is important in cell to cell communication [19].

GRP78 availability in these disease conditions might provide additional route of infection to COVID 19 as it might use GRP78 as the receptor for infecting cells overexpressing this receptor. Microsomal dissemination might also occur in case of IBD patients as in these patients GRP78 containing microsomes are expelled out from the intestinal cells. This can be a mode of cell to cell transmission of GRP loaded virus via microsomes.

\section{Perspective}

We still do not have specific antivirals or vaccine to fight COVID 19 which continues to affect the world health and economy. We don't understand this virus well and are in the process of understanding its nature, transmission and possibilities of therapeutic intervention.

Since we don't have tools to specifically fight this viral disease, it is important to understand how to protect those individuals who are at greater risk of progressing towards aggressive form of COVID 19 infection. For the same the first step is to identify such high-risk groups. Older age groups, people who have diabetes or hypertension have been already kept under high risk groups and thus these individuals are been advised to be more careful. Most of the measures taken or research done have been based on the existing idea of ACE2 as the primary COVID 19 receptor. Here, we have tried to connect the recently published result of the second receptor of COVID 19 i.e. GRP78 with the knowledge about prevalence of GRP 78 in various disease conditions as compared to normal individuals. We could narrow down our search into two major groups of diseases: Cancer and Autoimmune Diseases (RA and IBD). We propose that these two diseases should be categorized as high-risk groups as in such patients GRP78 gets upregulated posing increased risk for virus infection and spread to various tissue systems. 


\section{Acknowledgements}

We would extend our sincere acknowledgements to CSIR and AcSIR.

\section{Conflicts of Interest}

Authors declare no conflict of interest

\section{References}

1. Zhou, P., Yang, X., Wang, X., Hu, B., Zhang, L., Zhang, W. et al. A pneumonia outbreak associated with a new coronavirus of probable bat origin. 2020.

2. Ibrahim, I. M., Abdelmalek, D. H., Elshahat, M. E., and Elfiky, A. A. (2020) COVID-19 spike-host cell receptor GRP78 binding site prediction. Journal of Infection. 80(5), 554-562

3. Kim, Y. K., Back, S. H., Rho, J., Lee, S. H., and Jang, S. K. (2001). La autoantigen enhances translation of BiP mRNA. Nucleic Acids Res. 29, 50095016.

4. Suzuki, C. K., Bonifacino, J. S., Lin, A. Y., Davis, M. M., and Klausner, R. D. (1991). Regulating the retention of T-cell receptor alpha chain variants within the endoplasmic reticulum: $\mathrm{Ca}(2+)$-dependent association with BiP. J. Cell Biol. 114, 189-205.

5. Pfaffenbach, K. T., and Lee, A. S. (2011). The critical role of GRP78 in physiological and pathologic stress. Curr. Opin. Cell Biol. 23, 150-156. doi: 10.1016/j.ceb.2010.09.007

6. Daneshmand S, Quek ML, Lin E, Lee C, Cote RJ, Hawes D, Cai J, Groshen S, Lieskovsky G, Skinner DG et al.: Glucose-regulated protein GRP78 is upregulated in prostate cancer and correlates with recurrence and survival. Hum Pathol 2007, 38:1547-1552.

7. Lee E, Nichols P, Spicer D, Groshen S, Yu MC, Lee AS: GRP78 as a novel predictor of responsiveness to chemotherapy in breast cancer. Cancer Res 2006, 66:7849-7853.

8. Lee E, Nichols P, Groshen S, Spicer D, Lee AS: GRP78 as potential predictor for breast cancer response to adjuvant taxane therapy. Int J Cancer 2011, 128:726-731

9. Zhuang L, Scolyer RA, Lee CS, McCarthy SW, Cooper WA, Zhang XD, Thompson JF, Hersey P: Expression of glucoseregulated stress protein GRP78 is related to progression of melanoma. Histopathology 2009, 54:462-470.

10. Pyrko P, Schonthal AH, Hofman FM, Chen TC, Lee AS: The unfolded protein response regulator GRP78/BiP as a novel target for increasing chemosensitivity in malignant gliomas. Cancer Res 2007, 67:9809-9816.

11. Virrey JJ, Dong D, Stiles C, Patterson JB, Pen L, Ni M, Schonthal AH, Chen TC, Hofman FM, Lee AS: Stress chaperone GRP78/BiP confers chemoresistance to tumor-associated endothelial cells. Mol Cancer Res 2008, 6:1268-1275.

12. Arap MA, Lahdenranta J, Mintz PJ, Hajitou A, Sarkis AS, Arap W, Pasqualini R: Cell surface expression of the stress response chaperone GRP78 enables tumor targeting by circulating ligands. Cancer Cell 2004, 6:275-284. 
13. Misra UK, Pizzo SV: Ligation of cell surface GRP78 with antibody directed against the $\mathrm{COOH}$-terminal domain of GRP78 suppresses Ras/MAPK and PI 3-kinase/AKT signalling while promoting caspase activation in human prostate cancer cells. Cancer Biol Ther 2010, 9:142-152.

14. Liu Y, Steiniger SC, Kim Y, Kaufmann GF, Felding-Habermann B, Janda KD: Mechanistic studies of a peptidic GRP78 ligand for cancer cell-specific drug delivery. Mol Pharm 2007, 4:435-447.

15. Gonzalez-Gronow M, Cuchacovich M, Llanos C, Urzua C, Gawdi G, Pizzo SV: Prostate cancer cell proliferation in vitro is modulated by antibodies against glucose-regulated protein 78 isolated from patient serum. Cancer Res 2006, 66:11424-11431.

16. Misra UK, Mowery Y, Kaczowka S, Pizzo SV: Ligation of cancer cell surface GRP78 with antibodies directed against its $\mathrm{COOH}$-terminal domain upregulates p53 activity and promotes apoptosis. Mol Cancer Ther 2009, 8:13501362.

17. McFarland BC, Stewart J Jr, Hamza A, Nordal R, Davidson DJ, Henkin J, Gladson CL: Plasminogen Kringle 5 induces apoptosis of brain microvessel endothelial cells: sensitization by radiation and requirement for GRP78 and LRP1. Cancer Res 2009, 69:5537-5545.

18. Lu M.C., Lai N.S., Yu H.C., Huang H.B., Hsieh S.C., Yu C.L. 2010. Anticitrullinated protein antibodies bind surface-expressed citrullinated Grp78 on monocyte/macrophages and stimulate tumor necrosis factor alpha production. Arthritis Rheum. 62:1213-1223 10.1002/art.27386

19. Li Z, Zhuang M, Zhang L, Zheng X, Yang P, Li Z. Acetylation modification regulates GRP78 secretion in colon cancer cells. Sci Rep. (2016) 6:30406. doi: $10.1038 /$ srep30406 